\title{
Extension of the fractional derivative operator of the Riemann-Liouville
}

\author{
Dumitru Baleanu ${ }^{\mathrm{a}, \mathrm{b}, *}$, Praveen Agarwal $^{\mathrm{c}, \mathrm{d}}$, Rakesh K. Parmare ${ }^{\mathrm{e}}$, Maysaa M. Alqurashi ${ }^{\mathrm{f}}$, Soheil Salahshour ${ }^{\mathrm{g}}$ \\ ${ }^{a}$ Department of Mathematics, Cankaya University, Ankara, Turkey. \\ ${ }^{b}$ Institute of Space Sciences, Magurele-Bucharest, Romania. \\ ${ }^{c}$ Department of Mathematics, Anand International College of Engineering, Jaipur-303012, Republic of India. \\ ${ }^{d}$ Department of Mathematics, University Putra Malaysia, 43400 UPM, Serdang, Selangor, Malaysia. \\ ${ }^{e}$ Department of Mathematics, Govt. College of Engineering and Technology, Bikaner-334004, Rajasthan, India. \\ ${ }^{f}$ Department of Mathematics, King Saud University, P. O. Box 22452, Riyadh 11495, Saudi Arabia. \\ ${ }^{g}$ Department of Computer Engineering, Mashhad Branch, IAU, Iran.
}

Communicated by A. Atangana

\begin{abstract}
By using the generalized beta function, we extend the fractional derivative operator of the Riemann-Liouville and discusses its properties. Moreover, we establish some relations to extended special functions of two and three variables via generating functions. (C) 2017 All rights reserved.
\end{abstract}

Keywords: Hypergeometric function of two and three variables, fractional derivative operator, generating functions, Mellin transform.

2010 MSC: 33C05, 33C15.

\section{Introduction and preliminaries}

The concept of extension of fractional operators attracts the attention of many researchers (see, [1]). The present investigation was motivated by above mentioned works. We start from the Riemann-Liouville (RL) fractional derivative operator $\mathcal{D}_{z}^{\mu}$ (the reader may check [16] and [4, p. 70 et seq.]):

$$
\mathcal{D}_{z}^{\mu}\{f(z)\}:= \begin{cases}\frac{1}{\Gamma(-\mu)} \int_{0}^{z}(z-t)^{-\mu-1} f(t) d t, & (\Re(\mu)<0), \\ \frac{d^{m}}{d z^{m}}\left\{\mathcal{D}_{z}^{\mu-m}\{f(z)\},,\right. & (m-1 \leqq \Re(\mu)<m(m \in \mathbb{N})) .\end{cases}
$$

We recall that

$$
\mathcal{D}_{z}^{\mu}\left\{z^{\lambda}\right\}=\frac{\Gamma(\lambda+1)}{\Gamma(\lambda-\mu+1)} z^{\lambda-\mu}, \quad(\Re(\lambda)>-1)
$$

\footnotetext{
${ }^{*}$ Corresponding author

Email addresses: dumitru@caankaya.edu.tr (Dumitru Baleanu), goyal .praveen2011@gmail.com (Praveen Agarwal), (Rakesh K. Parmar), maysaa@ksu.edu.sa (Maysaa M. Alqurashi), soheilsalahshour@yahoo.com (Soheil Salahshour) doi:10.22436/jnsa.010.06.06
} 
where the path of integration in (1.1) denotes a line in the complex $t$-plane starting from $t=0$ and ending at $t=z$. Various extensions and generalizations of the RL fractional derivative operator were reported by various researchers (see, e.g., [4, 7, 8, 13, 16, 20]). In particular, in [10] the authors introduced the extended Riemann-Liouville fractional derivative operator $D_{z}^{\mu, p}$, namely:

$$
\mathcal{D}_{z}^{\mu, p}\{f(z)\}=\left\{\begin{array}{lc}
\frac{1}{\Gamma(-\mu)} \int_{0}^{z}(z-t)^{-\mu-1} \quad \exp \left(-\frac{p z^{2}}{(z-t) t}\right) f(t) d t, \quad(\Re(\mu)<0), \\
\frac{d^{m}}{d z^{m}}\left\{\mathcal{D}_{z}^{\mu-m}\{f(z)\}\right\}, \quad(m-1 \leqq \Re(\mu)<m(m \in \mathbb{N})) .
\end{array}\right.
$$

Clearly, the special case of (1.3), when $p=0$, reduces immediately to RL fractional derivative (see, [18, 19]). Making use of the definition (1.3), they derived the following extension of the fractional derivative (1.2)

$$
\mathcal{D}_{z}^{\mu, p}\left\{z^{\lambda}\right\}=\frac{B_{p}(\lambda+1,-\mu ; p)}{\Gamma(-\mu)} z^{\lambda-\mu}, \quad(\mathfrak{R}(\lambda)>-1 ; \mathfrak{R}(\mu)<0) .
$$

Recently, Choi et al. [3] introduced the extended beta and hypergeometric function

$$
\begin{aligned}
& B(x, y ; p, q):=\int_{0}^{1} t^{x-1}(1-t)^{y-1} \exp \left(-\frac{p}{t}-\frac{q}{1-t}\right) d t \\
& (\min \{\mathfrak{R}(x), \mathfrak{R}(y)\}>0 ; \min \{\mathfrak{R}(p), \mathfrak{R}(q)\} \geqq 0),
\end{aligned}
$$

and

$$
\begin{gathered}
\mathrm{F}_{\mathrm{p}, \mathrm{q}}(\mathrm{a}, \mathrm{b} ; \mathrm{c} ; z):={ }_{2} \mathrm{~F}_{1 ; \mathrm{p}, \mathrm{q}}(\mathrm{a}, \mathrm{b} ; \mathrm{c} ; z)=\sum_{\mathrm{n}=0}^{\infty}(\mathrm{a})_{\mathrm{n}} \frac{\mathrm{B}(\mathrm{b}+\mathrm{n}, \mathrm{c}-\mathrm{b} ; \mathrm{p}, \mathrm{q})}{\mathrm{B}(\mathrm{b}, \mathrm{c}-\mathrm{b})} \frac{z^{\mathrm{n}}}{\mathrm{n} !}, \\
(\mathrm{p} \geqq 0, \mathrm{q} \geqq 0 ;|z|<1 ; \mathfrak{R}(\mathrm{c})>\mathfrak{R}(\mathrm{b})>0),
\end{gathered}
$$

respectively.

Motivated by certain above recent extensions of the Euler's beta, Gauss's hypergeometric, and RL fractional derivative operator, we extend RL fractional derivative operator by introducing two additional parameters $p$ and $q$ in the integrand of (1.1). Moreover, we establish some relations to extended special functions of two and three variables of Appell and Lauricella hypergeometric functions via generating functions. It is expected that various other applications of extended RL fractional derivative operator (3.1) introduced here, can be useful in the field of applied mathematics and non-linear sciences (see, for details, $[2,5,6,9])$ and other related ones).

\section{Extension of hypergeometric functions and integral representations}

By making use of (1.4), we consider another extensions of Appell's and the Lauricella functions of two and three variables.

Definition 2.1. The extension of the hypergeometric functions of two and three variables are defined as:

$$
\begin{aligned}
F_{1}(a, b, c ; d ; x, y ; p, q) & =\sum_{m, n=0}^{\infty}(b)_{m}(c)_{n} \frac{B_{p, q}(a+m+n, d-a)}{B(a, d-a)} \frac{x^{m}}{m !} \frac{y^{n}}{n !}, \\
(\mathfrak{R}(p)>0, \mathfrak{R}(q)>0 ;|x|<1,|y|<1), & \\
F_{2}(a, b, c ; d, e ; x, y ; p, q) & =\sum_{m, n=0}^{\infty}(a)_{m+n} \frac{B_{p, q}(b+m, d-b) B(q)}{B(b, d-b) B(c, e-c)}(c+n-c) \\
(\mathfrak{R}(p) & >0, \mathfrak{R}(q)>0 ;|x|+|y|<1),
\end{aligned}
$$




$$
\begin{aligned}
F_{D}^{3}(a, b, c, d ; e ; x, y, z ; p, q) & =\sum_{m, n, r=0}^{\infty} \frac{B_{p, q}(a+m+n+r, e-a)(b)_{m}(c)_{n}(d)_{r}}{B(a, e-a)} \frac{x^{m}}{m !} \frac{y^{n}}{n !} \frac{z^{r}}{r !}, \\
(\mathfrak{R}(p) & >0, \mathfrak{R}(q)>0 ;|x|<1,|y|<1,|z|<1) .
\end{aligned}
$$

Remark 2.2. For $p=q$, the above definitions are similar to the ones in [10] and for $p=0=q$ similar to [18]. Also, definition (2.1) is studied in [14].

Theorem 2.3. The following result holds true for (2.1):

$$
F_{1}(a, b, c ; d ; x, y ; p, q)=\frac{1}{B(a, d-a)} \int_{0}^{1} t^{a-1}(1-t)^{d-a-1}(1-x t)^{-b}(1-y t)^{-c} e^{\left(-\frac{p}{t}-\frac{q}{1-t}\right)} d t .
$$

Proof. To prove this result, we start by assuming that

$$
\mathfrak{I}=\int_{0}^{1} \mathrm{t}^{\mathrm{a}-1}(1-\mathrm{t})^{\mathrm{d}-\mathrm{a}-1}(1-x \mathrm{t})^{-\mathrm{b}}(1-\mathrm{yt})^{-\mathrm{c}} e^{\left(-\frac{\mathrm{p}}{\mathrm{t}}-\frac{\mathrm{q}}{1-\mathrm{t}}\right)} \mathrm{dt} .
$$

Applying the binomial series expansion for $(1-x t)^{-b}$ and $(1-y t)^{-c}$ and interchanging the order of summation and integration, we get

$$
\begin{aligned}
\mathfrak{I} & =\int_{0}^{1} t^{a-1}(1-t)^{d-a-1} e^{\left(-\frac{p}{t}-\frac{q}{1-t}\right)}\left\{\sum_{n=0}^{\infty}(b)_{n} \frac{(x t)^{n}}{n !} \sum_{m=0}^{\infty}(c)_{m} \frac{(y t)^{m}}{m !}\right\} d t \\
& =\sum_{n=0}^{\infty} \sum_{m=0}^{\infty}(b)_{n}(c)_{m}\left\{\int_{0}^{1} t^{a+m+n-1}(1-t)^{d-a-1} e^{\left(-\frac{p}{t}-\frac{q}{1-t}\right)} d t\right\} \frac{x^{n}}{n !} \frac{y^{m}}{m !},
\end{aligned}
$$

by applying (1.4) and (2.1), we get (2.4).

Theorem 2.4. For (2.2) we have the following integral:

$$
\begin{aligned}
F_{2}(a, b, c ; d, e ; x, y ; p, q) & =\frac{1}{B(b, d-b) B(c, e-c)} \\
& =\int_{0}^{1} \int_{0}^{1} \frac{t^{b-1}(1-t)^{d-b-1} s^{c-1}(1-s)^{e-c-1}}{(1-x t-y s)^{a}} e^{\left(-\frac{p}{t}-\frac{q}{1-t}-\frac{p}{s}-\frac{q}{1-s}\right)} d t d s
\end{aligned}
$$

Proof. We start by expanding $(1-x t-y s)^{-a}$; we have

$$
\begin{aligned}
& \int_{0}^{1} \int_{0}^{1} \frac{t^{b-1}(1-t)^{d-b-1} s^{c-1}(1-s)^{e-c-1}}{(1-x t-y s)^{a}} e^{\left(-\frac{p}{t}-\frac{q}{1-t}-\frac{p}{s}-\frac{q}{1-s}\right)} d t d s \\
& \quad=\int_{0}^{1} \int_{0}^{1} t^{b-1}(1-t)^{d-b-1} e^{\left(-\frac{p}{t}-\frac{q}{1-t}\right)} s^{c-1}(1-s)^{e-c-1} e^{\left(-\frac{p}{s}-\frac{q}{1-s}\right)} \sum_{N=0}^{\infty}(a)_{N} \frac{(x t+y s)^{N}}{N !} d t d s .
\end{aligned}
$$

Using the series identity

$$
\sum_{N=0}^{\infty} f(N) \frac{(x+y)^{N}}{N !}=\sum_{r=0}^{\infty} \sum_{l=0}^{\infty} f(l+r) \frac{x^{r}}{r !} \frac{y^{l}}{l !}
$$

we get

$$
\begin{aligned}
& \int_{0}^{1} \int_{0}^{1} \frac{t^{b-1}(1-t)^{d-b-1} s^{c-1}(1-s)^{e-c-1}}{(1-x t-y s)^{a}} e^{\left(-\frac{p}{t}-\frac{q}{1-t}-\frac{p}{s}-\frac{q}{1-s}\right)} d t d s \\
& \quad=\int_{0}^{1} \int_{0}^{1} t^{b-1}(1-t)^{d-b-1} e^{\left(-\frac{p}{t}-\frac{q}{1-t}\right)} s^{c-1}(1-s)^{e-c-1} e^{\left(-\frac{p}{s}-\frac{q}{1-s}\right)} \times \sum_{r=0}^{\infty} \sum_{l=0}^{\infty}(a)_{r+l} \frac{(x t)^{r}}{r !} \frac{(y s)^{l}}{l !} d t d s .
\end{aligned}
$$


Here, involving series and the integrals are convergent, then by interchanging the order of summation and integration, we obtain

$$
\begin{aligned}
& \int_{0}^{1} \int_{0}^{1} \frac{t^{b-1}(1-t)^{d-b-1} s^{c-1}(1-s)^{e-c-1}}{(1-x t-y s)^{a}} e^{\left(-\frac{p}{t}-\frac{q}{1-t}-\frac{p}{s}-\frac{q}{1-s}\right)} d t d s \\
& \quad=\sum_{r=0}^{\infty} \sum_{l=0}^{\infty}(a)_{l+r} \frac{x^{r}}{r !} \frac{y^{l}}{l !} \int_{0}^{1} t^{b+r-1}(1-t)^{d-b-1} e^{\left(-\frac{p}{t}-\frac{q}{1-t}\right)} d t \int_{0}^{1} s^{c+l-1}(1-s)^{e-c-1} e^{\left(-\frac{p}{s}-\frac{q}{1-s}\right)} d s,
\end{aligned}
$$

by applying (1.4) and (2.2), we get (2.5).

Theorem 2.5. For (2.3) the following integral holds true

$$
\mathrm{F}_{\mathrm{D}}^{3}(\mathrm{a}, \mathrm{b}, \mathrm{c}, \mathrm{d} ; \mathrm{e} ; \mathrm{x}, \mathrm{y}, z ; \mathrm{p}, \mathrm{q})=\frac{\Gamma(e)}{\Gamma(\mathrm{a}) \Gamma(e-\mathrm{a})} \int_{0}^{1} \mathrm{t}^{\mathrm{a}-1}(1-\mathrm{t})^{\mathrm{e}-\mathrm{a}-1}(1-\mathrm{xt})^{-\mathrm{b}}(1-\mathrm{yt})^{-\mathrm{c}}(1-\mathrm{yt})^{-\mathrm{d}} e^{\left(-\frac{\mathrm{p}}{\mathrm{t}}-\frac{\mathrm{q}}{1-\mathrm{t}}\right)} \mathrm{dt} .
$$

Proof. This proof looks similar to the one presented in Theorem 2.3.

\section{Extension of RL fractional derivative operator}

Now, we introduce new extension of the RL type fractional derivative operator.

Definition 3.1. The extended RL type fractional derivative operator is defined by

$$
\mathcal{D}_{z}^{\eta}\{f(z) ; p, q\}:= \begin{cases}\frac{1}{\Gamma(-\eta)} \int_{0}^{z}(z-t)^{-\eta-1} e^{\left(\frac{-p z}{t}-\frac{q z}{z-t}\right)} f(t) d t, & (\Re(\eta)<0), \\ \frac{d^{m}}{d z^{m}}\left\{\mathcal{D}_{z}^{\eta-m}\{f(z) ; p, q\}\right\}, & (m-1 \leqq \Re(\eta)<m(m \in \mathbb{N})),\end{cases}
$$

where $\mathfrak{R}(p)>0, \mathfrak{R}(q)>0$ and the path of integration is a line from 0 to $z$ in the complex $t$-plane.

Clearly for $p=q,(3.1)$ reduces to (1.3) and for $p=0=q$, we obtain its classical form (see, for details $[4,18,19])$.

Now, we establish some theorems involving the extended fractional derivatives.

Theorem 3.2. The following representation for (3.1) is valid:

$$
\mathrm{D}_{z}^{\eta}\left[z^{\lambda} ; p, q\right]=\frac{\mathrm{B}_{\mathrm{p}, \mathrm{q}}(\lambda+1,-\eta)}{\Gamma(-\eta)} z^{\lambda-\eta} \quad(\operatorname{Re}(\eta)<0) .
$$

Proof. Using (3.1) and (1.4), we get

$$
D_{z}^{\eta}\left[z^{\lambda} ; p, q\right]=\frac{1}{\Gamma(-\eta)} \int_{0}^{z} t^{\lambda}(z-t)^{-\eta-1} e^{\left(-\frac{p z}{t}-\frac{q z}{z-t}\right)} d t
$$

replacing $t=u z$, we have

$$
\begin{aligned}
D_{z}^{\eta}\left[z^{\lambda} ; p, q\right] & =\frac{1}{\Gamma(-\eta)} \int_{0}^{1}(u z)^{\lambda}(z-u z)^{-\eta-1} e^{\left(-\frac{p z}{u z}-\frac{q}{z-u z}\right)} z d u \\
& =\frac{z^{\lambda-\eta}}{\Gamma(-\eta)} \int_{0}^{1} u^{\lambda}(1-u)^{-\eta-1} e^{\left(-\frac{p}{u}-\frac{q}{1-u}\right)} d u .
\end{aligned}
$$

By applying definition (1.4) we yield (3.2) directly. 
Theorem 3.3. Let $\mathfrak{R}(\eta)<0$ and suppose that a function $\mathrm{f}(\boldsymbol{z})$ is analytic at the origin with its Maclaurin expansion given by $f(z)=\sum_{n=0}^{\infty} a_{n} z^{n}(|z|<\rho)$ for some $\rho \in \mathbb{R}^{+}$. Then we have

$$
D_{z}^{\eta}[f(z) ; p, q]=\sum_{n=0}^{\infty} a_{n} D_{z}^{\eta}\left[z^{n} ; p, q\right] .
$$

Proof. We begin from Definition 3.1 to the function $f(z)$ with its series expansion and we conclude

$$
D_{z}^{\eta}[f(z) ; p, q]=\frac{1}{\Gamma(-\eta)} \int_{0}^{z} \sum_{n=0}^{\infty} a_{n} t^{n}(z-t)^{-\eta-1} e^{\left(-\frac{p z}{t}-\frac{q z}{z-t}\right)} d t
$$

Since the series is uniformly convergent on any closed disk centered at the origin with its radius smaller than $\rho$, so does the series on the line segment from 0 to a fixed $z$ for $|z|<\rho$. This fact guarantees term-by-term integration as follows:

$$
D_{z}^{\eta}[f(z) ; p, q]=\sum_{n=0}^{\infty} a_{n}\left\{\frac{1}{\Gamma(-\eta)} \int_{0}^{z} t^{n}(z-t)^{-\eta-1} e^{\left(-\frac{p z}{t}-\frac{q z}{z-t}\right)} d t\right\}=\sum_{n=0}^{\infty} a_{n} D_{z}^{\eta}\left[z^{n} ; p, q\right] .
$$

So, the proof is completed.

Theorem 3.4. The following result holds true:

$$
\begin{aligned}
\mathrm{D}_{z}^{\lambda-\eta}\left[z^{\lambda-1}(1-z)^{-\alpha} ; \mathrm{p}, \mathrm{q}\right] & =\frac{\Gamma(\lambda)}{\Gamma(\eta)} z^{\eta-1}{ }_{2} \mathrm{~F}_{1 ; p, q}(\alpha, \lambda ; \eta ; z), \\
(\mathfrak{R}(\eta)>\mathfrak{R}(\lambda) & >0, \text { and }|z|<1) .
\end{aligned}
$$

Proof. Some direct calculations yield

$$
\begin{aligned}
D_{z}^{\lambda-\eta}\left[z^{\lambda-1}(1-z)^{-\alpha} ; p, q\right] & =\frac{1}{\Gamma(\eta-\lambda)} \int_{0}^{z} t^{\lambda-1}(1-t)^{-\alpha} e^{\left(-\frac{p z}{t}-\frac{q z}{z-t}\right)}(z-t)^{\eta-\lambda-1} d t \\
& =\frac{z^{\eta-\lambda-1}}{\Gamma(\eta-\lambda)} \int_{0}^{z} t^{\lambda-1}(1-t)^{-\alpha}\left(1-\frac{t}{z}\right)^{\eta-\lambda-1} e^{\left(-\frac{p z}{t}-\frac{q z}{z-t}\right)} d t \\
& =\frac{z^{\eta-\lambda-1} z^{\lambda}}{\Gamma(\eta-\lambda)} \int_{0}^{1} u^{\lambda-1}(1-u z)^{-\alpha}(1-u)^{\eta-\lambda-1} e^{\left(-\frac{p}{u}-\frac{q}{1-u}\right)} d u
\end{aligned}
$$

Using (1.5) and after little simplification, we have the (3.3). In this way we finished the proof.

Theorem 3.5. The representation given below holds true:

$$
\begin{aligned}
& \mathrm{D}_{z}^{\lambda-\eta}\left[z^{\lambda-1}(1-\mathrm{a} z)^{-\alpha}(1-\mathrm{b} z)^{-\beta} ; \mathrm{p}, \mathrm{q}\right]=\frac{\Gamma(\lambda)}{\Gamma(\eta)} z^{\eta-1} \mathrm{~F}_{1}(\lambda, \alpha, \beta ; \eta ; \mathrm{a} z, \mathrm{~b} z ; \mathrm{p}, \mathrm{q}), \\
& (\mathfrak{R}(\alpha)>0, \mathfrak{R}(\beta)>0, \mathfrak{R}(\eta)>\mathfrak{R}(\lambda)>0 ;|\mathrm{a} z|<1, \text { and }|\mathrm{b} z|<1) .
\end{aligned}
$$

More generally, we have

$$
\begin{aligned}
& \mathrm{D}_{z}^{\lambda-\eta}\left[z^{\lambda-1}(1-\mathrm{a} z)^{-\alpha}(1-\mathrm{b} z)^{-\beta}(1-\mathrm{c} z)^{-\gamma} ; \mathrm{p}, \mathrm{q}\right]=\frac{\Gamma(\lambda)}{\Gamma(\eta)} z^{\eta-1} \mathrm{~F}_{\mathrm{D}}^{3}(\lambda, \alpha, \beta, \gamma ; \eta ; \mathrm{a} z, \mathrm{~b} z, \mathrm{c} z ; \mathrm{p}, \mathrm{q}), \\
& (\mathfrak{R}(\alpha)>0, \mathfrak{R}(\beta)>0, \mathfrak{R}(\gamma)>0, \mathfrak{R}(\eta)>\mathfrak{R}(\lambda)>0,|\mathrm{a} z|<1,|\mathrm{~b} z|<1, \text { and }|\mathrm{c} z|<1) .
\end{aligned}
$$


Proof. To prove (3.4), using the following power series expansion for $(1-a z)^{-\alpha}$ and $(1-b z)^{-\beta}$

$$
(1-a z)^{-\alpha}(1-b z)^{-\beta}=\sum_{l=0}^{\infty} \sum_{k=0}^{\infty}(\alpha)_{l}(\beta)_{k} \frac{(a z)^{l}}{l !} \frac{(b z)^{k}}{k !}
$$

then applying Theorem 3.2, we conclude that

$$
\begin{aligned}
D_{z}^{\lambda-\eta}\left[z^{\lambda-1}(1-a z)^{-\alpha}(1-b z)^{-\beta} ; p, q\right] & =\sum_{l=0}^{\infty} \sum_{k=0}^{\infty}(\alpha)_{l}(\beta)_{k} \frac{(a)^{l}}{l !} \frac{(b)^{k}}{k !} D_{z}^{\lambda-\eta}\left[z^{\lambda+l+k-1} ; p, q\right] \\
& =\sum_{l=0}^{\infty} \sum_{k=0}^{\infty}(\alpha)_{l}(\beta)_{k} \frac{(a)^{l}}{l !} \frac{(b)^{k}}{k !} \frac{B_{p, q}(\lambda+l+k, \eta-\lambda)}{\Gamma(\eta-\lambda)} z^{l+k+\eta-1} .
\end{aligned}
$$

Now, applying (2.1), we obtain

$$
\mathrm{D}_{z}^{\lambda-\eta}\left[z^{\lambda-1}(1-\mathrm{a} z)^{-\alpha}(1-\mathrm{b} z)^{-\beta} ; \mathrm{p}, \mathrm{q}\right]=\frac{\Gamma(\lambda)}{\Gamma(\eta)} z^{\eta-1} \mathrm{~F}_{1}(\lambda, \alpha, \beta ; \eta ; \mathrm{a} z, \mathrm{~b} z ; \mathrm{p}, \mathrm{q}) .
$$

Similarly, as in the proof of (3.4), taking the binomial theorem for $(1-a z)^{-\alpha},(1-b z)^{-\beta}$, and $(1-c z)^{-\gamma}$, then applying Theorem 3.2 and (2.3) into account, one can easily prove (3.5). Therefore, we omit the proof.

Theorem 3.6. The representation written below holds true:

$$
\begin{aligned}
& \mathrm{D}_{z}^{\lambda-\eta}\left[z^{\lambda-1}(1-z)^{-\alpha} \mathrm{F}_{\mathrm{p}, \mathrm{q}}\left(\alpha, \beta ; \gamma ; \frac{x}{1-z}\right) ; \mathrm{p}, \mathrm{q}\right]=\frac{1}{\mathrm{~B}(\beta, \gamma-\beta) \Gamma(\eta-\lambda)} z^{\eta-1} \mathrm{~F}_{2}(\alpha, \beta, \lambda ; \gamma, \eta ; x, z ; \mathrm{p}, \mathrm{q}), \\
& \left(\mathfrak{R}(\alpha)>0, \mathfrak{R}(\beta)>0, \mathfrak{R}(\gamma)>0, \mathfrak{R}(\mu)>\mathfrak{R}(\lambda)>0, ;\left|\frac{x}{1-z}\right|<1, \text { and }|x|+|z|<1\right) .
\end{aligned}
$$

Proof. Applying (2.2) on the LHS of (3.6), we conclude

$$
\begin{aligned}
D_{z}^{\lambda-\eta}\left[z^{\lambda-1}(1-z)^{-\alpha} F_{p, q}\left(\alpha, \beta ; \gamma ; \frac{x}{1-z}\right) ; p, q\right] \\
\quad=D_{z}^{\lambda-\eta}\left[z^{\lambda-1}(1-z)^{-\alpha}\left\{\sum_{n=0}^{\infty} \frac{(\alpha)_{n} B_{p, q}(\beta+n, \gamma-\beta)}{B(\beta, \gamma-\beta) n !}\left(\frac{x}{1-z}\right)^{n}\right\} ; p, q\right] \\
\quad=\frac{1}{B(\beta, \gamma-\beta)} D_{z}^{\lambda-\eta}\left[z^{\lambda-1} \sum_{n=0}^{\infty}(\alpha)_{n} B_{p, q}(\beta+n, \gamma-\beta) \frac{x^{n}}{n !}\left\{(1-z)^{-\alpha-n}\right\} ; p, q\right] .
\end{aligned}
$$

Using the power series expansion for $(1-z)^{-\alpha-n}$, applying Theorem 3.2 and (2.2), we conclude that

$$
\begin{aligned}
& D_{z}^{\lambda-\eta}\left[z^{\lambda-1}(1-z)^{-\alpha} F_{p, q}\left(\alpha, \beta ; \gamma ; \frac{x}{1-z}\right) ; p, q\right] \\
& \quad=D_{z}^{\lambda-\eta}\left[z^{\lambda-1}(1-z)^{-\alpha}\left\{\sum_{n=0}^{\infty} \frac{(\alpha)_{n} B_{p, q}(\beta+n, \gamma-\beta)}{B(\beta, \gamma-\beta) n !}\left(\frac{x}{1-z}\right)^{n}\right\} ; p, q\right] \\
& =\frac{1}{B(\beta, \gamma-\beta)} D_{z}^{\lambda-\eta}\left[z^{\lambda-1} \sum_{n=0}^{\infty}(\alpha)_{n} B_{p, q}(\beta+n, \gamma-\beta) \frac{x^{n}}{n !}\left\{(1-z)^{-\alpha-n}\right\} ; p, q\right] .
\end{aligned}
$$

This ends the proof.

\section{Mellin transform representations}

The double Mellin transforms [12, p. 293, Eq. (7.1.6)] of a integrable function $f(x, y)$ with indices $r$ and $s$ is defined by

$$
\mathfrak{M}\{f(x, y): x \rightarrow r, y \rightarrow s\}:=\int_{0}^{\infty} \int_{0}^{\infty} x^{r-1} y^{s-1} f(x, y) d x d y
$$


Theorem 4.1. The following Mellin transform formula is valid:

$$
\begin{gathered}
\mathfrak{M}\left\{\mathrm{D}_{z}^{\mu, p, q}\left(z^{\lambda}\right): p \rightarrow r, q \rightarrow s\right\}:=\frac{\Gamma(r) \Gamma(s)}{\Gamma(-\mu)} \mathrm{B}(\lambda+r+1, s-\mu) z^{\lambda-\mu}, \\
(\mathfrak{R}(\lambda)>-1, \mathfrak{R}(\mu)<0, \mathfrak{R}(s)>0, \mathfrak{R}(r)>0) .
\end{gathered}
$$

Proof. Applying the definition (4.1) on (3.1), we get

$$
\begin{aligned}
\mathfrak{M} & \left\{D_{z}^{\mu, p, q}\left(z^{\lambda}\right): p \rightarrow r, q \rightarrow s\right\} \\
& :=\int_{0}^{\infty} \int_{0}^{\infty} p^{r-1} q^{s-1} D_{z}^{\mu, p, q}\left(z^{\lambda}\right) d p d q \\
& =\frac{1}{\Gamma(-\mu)} \int_{0}^{\infty} \int_{0}^{\infty} p^{r-1} q^{s-1}\left[\int_{0}^{z} t^{\lambda}(z-t)^{-\mu-1} \exp \left(-\frac{p z}{t}-\frac{q z}{z-t}\right) d t\right] d p d q \\
& =\frac{z^{-\mu-1}}{\Gamma(-\mu)} \int_{0}^{\infty} \int_{0}^{\infty} p^{r-1} q^{s-1}\left[\int_{0}^{z} t^{\lambda}\left(1-\frac{t}{z}\right)^{-\mu-1} \exp \left(-\frac{p z}{t}-\frac{q z}{z-t}\right) d t\right] d p d q \\
& =\frac{z^{-\mu-1}}{\Gamma(-\mu)} \int_{0}^{\infty} \int_{0}^{\infty} p^{r-1} q^{s-1}\left[\int_{0}^{1} u^{\lambda} z^{\lambda}(1-u)^{-\mu-1} \exp \left(-\frac{p}{u}-\frac{q}{1-u}\right) d t\right] d p d q \\
& =\frac{z^{\lambda-\mu}}{\Gamma(-\mu)} \int_{0}^{\infty} \int_{0}^{\infty} p^{r-1} q^{s-1}\left[\int_{0}^{1} u^{\lambda}(1-u)^{-\mu-1} \exp \left(-\frac{p}{u}-\frac{q}{1-u}\right) d t\right] d p d q \\
& =\frac{z^{\lambda-\mu}}{\Gamma(-\mu)} \int_{0}^{1} u^{\lambda}(1-u)^{-\mu-1}\left(\int_{0}^{\infty} p^{r-1} \exp \left(\frac{-p}{u}\right) d p\right)\left(\int_{0}^{\infty} q^{s-1} \exp \left(\frac{-q}{1-u}\right) d q\right) d u,
\end{aligned}
$$

where we have changed the order of integration by absolutely convergent under the stated conditions. Using the definition of Gamma function, we have

$$
\begin{aligned}
\mathcal{M}\left\{D_{z}^{\mu, p, q}\left(z^{\lambda}\right): p \rightarrow r, q \rightarrow s\right\} & :=\frac{z^{\lambda-\mu}}{\Gamma(-\mu)} \int_{0}^{1} u^{\lambda}(1-u)^{-\mu-1} u^{r} \Gamma(r)(1-u)^{s} \Gamma(s) d u \\
& =\frac{z^{\lambda-\mu} \Gamma(r) \Gamma(s)}{\Gamma(-\mu)} \int_{0}^{1} u^{\lambda+r}(1-u)^{s-\mu-1} d u=\frac{z^{\lambda-\mu} \Gamma(r) \Gamma(s)}{\Gamma(-\mu)} B(\lambda+r+1, s-\mu),
\end{aligned}
$$

which completes the proof.

Theorem 4.2. The following formula holds true:

$$
\begin{aligned}
& \mathfrak{M}\left\{\mathrm{D}_{z}^{\mu, p, q}\left((1-z)^{-\alpha}\right):\right.p \rightarrow r, \mathrm{q} \rightarrow s\}:=\frac{\Gamma(\mathrm{r}) \Gamma(s) z^{-\mu}}{\Gamma(-\mu) \mathrm{B}(\mathrm{r}+1, \mathrm{~s}-\mu)} \mathrm{F}(\alpha, \mathrm{r}+1 ; \mathrm{r}+\mathrm{s}-\mu+1 ; z), \\
&(\mathfrak{R}(\mu)<0, \mathfrak{R}(\mathrm{s})>0, \mathfrak{R}(\mathrm{r})>0, \mathfrak{R}(\alpha)>0, \text { and }|z|<1) .
\end{aligned}
$$

Proof. Applying Theorem 4.1 with $\lambda=n$, we can write that

$$
\begin{aligned}
\mathfrak{M}\left\{D_{z}^{\mu, p, q}\left((1-z)^{-\alpha}\right): p \rightarrow r, q \rightarrow s\right\} & :=\sum_{n=0}^{\infty} \frac{(\alpha)_{n}}{n !} \mathfrak{M}\left\{D_{z}^{\mu, p, q}\left((1-z)^{-\alpha}\right): p \rightarrow r, q \rightarrow s\right\} \\
& =\frac{\Gamma(r) \Gamma(s)}{\Gamma(-\mu)} \sum_{n=0}^{\infty} \frac{(\alpha)_{n}}{n !} B(n+r+1, s-\mu) z^{n-\mu}
\end{aligned}
$$




$$
\begin{aligned}
& =\frac{\Gamma(r) \Gamma(s) z^{-\mu}}{\Gamma(-\mu)} \sum_{n=0}^{\infty} B(n+r+1, s-\mu) \frac{(\alpha)_{n} z^{n}}{n !} \\
& =\frac{\Gamma(r) \Gamma(s) z^{-\mu}}{\Gamma(-\mu) B(r+1, s-\mu)} F(\alpha, r+1 ; r+s-\mu+1 ; z) .
\end{aligned}
$$

This completes the proof.

\section{Generating relations and further results}

Here, we obtain some generating relations of linear and bilinear type for the extended hypergeometric functions.

Theorem 5.1. The below generating relation is valid:

$$
\begin{aligned}
& \sum_{n=0}^{\infty} \frac{(\lambda)_{n}}{n !}{ }_{2} F_{1 ; p, q}(\lambda+n, \alpha ; \beta ; x) t^{n}=(1-t)_{2}^{-\lambda} F_{1 ; p, q}\left(\lambda, \alpha ; \beta ; \frac{x}{1-t}\right), \\
& \quad(|x|<\min (1,|1-t|) \text { and } \mathfrak{R}(\lambda)>0, \mathfrak{R}(\beta)>\mathfrak{R}(\alpha)>0) .
\end{aligned}
$$

Proof. Let us consider the series identity

$$
[(1-x)-t]^{-\lambda}=(1-t)^{-\lambda}\left[1-\frac{x}{1-t}\right]^{-\lambda}
$$

Using the power series expansion, we have

$$
\sum_{n=0}^{\infty} \frac{(\lambda)_{n}}{n !}(1-x)^{-\lambda}\left(\frac{t}{1-x}\right)^{n}=(1-t)^{-\lambda}\left[1-\frac{x}{1-t}\right]^{-\lambda}
$$

By using the multiplication of both sides by $x^{\alpha-1}$ followed by applying the operator $D_{x}^{\alpha-\beta, p, q}$, we conclude that

$$
D_{x}^{\alpha-\beta, p, q}\left[\sum_{n=0}^{\infty} \frac{(\lambda)_{n}}{n !}(1-x)^{-\lambda}\left(\frac{t}{1-x}\right)^{n} x^{\alpha-1}\right]=(1-t)^{-\lambda} D_{x}^{\alpha-\beta, p, q}\left[x^{\alpha-1}\left(1-\frac{x}{1-t}\right)^{-\lambda}\right] .
$$

Again, by utilizing the interchange of the order, which holds true under the stated conditions, we conclude

$$
\sum_{n=0}^{\infty} \frac{(\lambda)_{n}}{n !} D_{x}^{\alpha-\beta, p, q}\left[x^{\alpha-1}(1-x)^{-\lambda-n}\right] t^{n}=(1-t)^{-\lambda} D_{x}^{\alpha-\beta, p, q}\left[x^{\alpha-1}\left(1-\frac{x}{1-t}\right)^{-\lambda}\right] .
$$

Using Theorem 3.3, the result is reported.

Theorem 5.2. Following generating relation holds true:

$$
\begin{gathered}
\sum_{n=0}^{\infty} \frac{(\lambda)_{n}}{n !}{ }_{2} F_{1 ; p, q}(\rho-n, \alpha ; \beta ; x) t^{n}=(1-t)^{-\lambda} F_{1}\left(\alpha, \rho, \lambda ; \beta ; x, \frac{-x t}{1-t} ; p, q\right), \\
\left(\Re(\beta)>\mathfrak{R}(\alpha)>0, \mathfrak{R}(\rho)>0, \mathfrak{R}(\lambda)>0 ;|t|<\frac{1}{1+|x|} .\right.
\end{gathered}
$$

Proof. To prove the above theorem we use the series identity

$$
[1-(1-x) t]^{-\lambda}=(1-t)^{-\lambda}\left[1+\frac{x t}{1-t}\right]^{-\lambda}
$$


Using the series expansion to the left hand side, we have

$$
\sum_{n=0}^{\infty} \frac{(\lambda)_{n}}{n !}(1-x)^{n} t^{n}=(1-t)^{-\lambda}\left[1-\frac{-x t}{1-t}\right]^{-\lambda}
$$

After the multiplication of the both sides by $x^{\alpha-1}(1-x)^{-\rho}$ and by utilizing the operator $D_{x}^{\alpha-\beta, p, q}$ on both sides, we conclude

$$
D_{x}^{\alpha-\beta, p, q}\left[\sum_{n=0}^{\infty} \frac{(\lambda)_{n}}{n !} x^{\alpha-1}(1-x)^{-\rho+n} t^{n}\right]=(1-t)^{-\lambda} D_{x}^{\alpha-\beta, p, q}\left[x^{\alpha-1}(1-x)^{-\rho}\left(\left(1-\frac{-\chi t}{1-t}\right)^{-\lambda}\right] .\right.
$$

Again by changing the order, valid for $\mathfrak{R}(\alpha)>0$ and $|x t|<|1-t|$, we end up with a conclusion that

$$
\sum_{n=0}^{\infty} \frac{(\lambda)_{n}}{n !} D_{x}^{\alpha-\beta, p, q}\left[x^{\alpha-1}(1-x)^{-\rho+n}\right] t^{n}=(1-t)^{-\lambda} D_{x}^{\alpha-\beta, p, q}\left[x^{\alpha-1}(1-x)^{-\rho}\left(\left(1-\frac{-x t}{1-t}\right)^{-\lambda}\right] .\right.
$$

Using Theorem 3.3, we obtain the required theorem.

Theorem 5.3. The following bilinear generating relation holds true:

$$
\begin{gathered}
\sum_{n=0}^{\infty} \frac{(\lambda)_{n}}{n !}{ }_{2} F_{1 ; p, q}(\gamma,-n ; \delta ; y){ }_{2} F_{1 ; p, q}(\lambda+n, \alpha ; \beta ; x) t^{n}=(1-t)^{-\lambda} F_{2}\left(\lambda, \alpha, \gamma ; \beta, \delta ; \frac{x}{1-t}, \frac{-y t}{1-t} ; p, q\right) \\
\left(\mathfrak{R}(\delta)>\Re(\gamma)>0, \mathfrak{R}(\alpha)>0, \mathfrak{R}(\lambda)>0, \mathfrak{R}(\beta)>0 ;|t|<\frac{1-|x|}{1+|y|} \text { and }|x|<1\right) .
\end{gathered}
$$

Proof. Replacing $\mathrm{t} \rightarrow(1-\mathrm{y}) \mathrm{t}$ in Theorem 5.1, multiplying $\mathrm{y}^{\gamma-1}$ to the resulting equality and then using the operator $D_{y}^{\gamma-\delta, p, q}$, we get

$$
\begin{aligned}
& D_{y}^{\gamma-\delta, p, q}\left[\sum_{n=0}^{\infty} \frac{(\lambda)_{n}}{n !} y_{2}^{\gamma-1} F_{1 ; p, q}(\lambda+n, \alpha ; \beta ; x)(1-y)^{n} t^{n}\right] \\
& \quad=D_{y}^{\gamma-\delta, p, q}\left[(1-(1-y) t)^{-\lambda} y_{2}^{\gamma-1} F_{1 ; p, q}\left(\lambda, \alpha ; \beta ; \frac{x}{1-(1-y) t}\right)\right] .
\end{aligned}
$$

Once again we are allowed to interchange the order and we conclude that

$$
\begin{aligned}
& \sum_{n=0}^{\infty} \frac{(\lambda)_{n}}{n !} D_{y}^{\gamma-\delta, p, q}\left[y^{\gamma-1}(1-y)^{n}\right]_{2} F_{1 ; p, q}(\lambda+n, \alpha ; \beta ; x) t^{n} \\
& \quad=(1-t)^{-\lambda} D_{y}^{\gamma-\delta, p, q}\left[y^{\gamma-1}\left(1-\frac{-y t}{1-t}\right)_{2}^{-\lambda} F_{1 ; p, q}\left(\lambda, \alpha ; \beta ; \frac{\frac{x}{1-t}}{1-\frac{-y t}{1-t}}\right)\right] .
\end{aligned}
$$

Using Theorems 3.3 and 3.4, we proved the result.

Remark 5.4. For $p=0=q$, the obtained results reduce to the corresponding known results (see, for details, $[4,16,18,19])$.

Theorem 5.5. Let $\mathfrak{R}(p)>0, \mathfrak{R}(q)>0, \mathfrak{R}(\mu)>\mathfrak{R}(\lambda)>0 ; \gamma, \delta \in \mathbb{C}$ and the extended Riemann-Liouville fractional derivative (3.1). Then we conclude that:

$$
\mathrm{D}_{z}^{\lambda-\mu, p, q}\left[z^{\lambda-1} E_{\gamma, \delta}^{\mu}(z)\right]=\frac{z^{\mu-1}}{\Gamma(\mu-\lambda)} \sum_{n=0}^{\infty} \frac{(\mu)_{n}}{\Gamma(\gamma n+\delta)} B_{p, q}(\lambda+n, \mu-\lambda) \frac{z^{n}}{n !},
$$

where $\mathrm{E}_{\gamma, \delta}^{\mu}(z)$ denotes generalized Mittag-Leffler function [15] written as:

$$
E_{\gamma, \delta}^{\mu}(z)=\sum_{n=0}^{\infty} \frac{(\mu)_{n}}{\Gamma(\gamma n+\delta)} \frac{z^{n}}{n !}, \quad(\gamma, \delta, \mu \in \mathbb{C} ; \mathfrak{R}(\gamma)>0) .
$$


Proof. Applying (5.2) to (5.1) and using Theorems 3.6 and 3.2, we get

$$
\begin{aligned}
\mathrm{D}_{z}^{\lambda-\mu, p, q}\left[z^{\lambda-1} \mathrm{E}_{\gamma, \delta}^{\mu}(z)\right] & =\mathrm{D}_{z}^{\lambda-\mu, p, q}\left[z^{\lambda-1}\left\{\sum_{n=0}^{\infty} \frac{(\mu)_{n}}{\Gamma(\gamma n+\delta)} \frac{z^{n}}{n !}\right\}\right] \\
& =\sum_{n=0}^{\infty} \frac{(\mu)_{n}}{\Gamma(\gamma n+\delta) n !}\left\{D_{z}^{\lambda-\mu, p, q}\left[z^{\lambda+n-1}\right]\right\} \\
& =\sum_{n=0}^{\infty} \frac{(\mu)_{n}}{\Gamma(\gamma n+\delta) n !}\left\{\frac{B_{p, q}(\lambda+n, \mu-\lambda)}{\Gamma(\mu-\lambda)} z^{\mu+n-1}\right\} .
\end{aligned}
$$

Remark 5.6. If we set $\mathrm{p}=\mathrm{q}$ in (5.1), we get the interesting known result given by Özarslan and Yilmaz [11, Theorem 9].

Theorem 5.7. Let $\mathfrak{R}(p)>0, \mathfrak{R}(q)>0, \mathfrak{R}(\mu)>\mathfrak{R}(\lambda)>0 ; \gamma, \delta \in \mathbb{C}$ and the extended Riemann-Liouville fractional derivative (3.1). Then we conclude that:

$$
D_{z}^{\lambda-\mu, p, q}\left[z^{\lambda-1}{ }_{m} \Psi_{n}\left[z \mid \begin{array}{c}
\left(a_{i}, \alpha_{i}\right)_{1, m} \\
\left(b_{j}, \beta_{j}\right)_{1, n}
\end{array}\right]\right]=\frac{z^{\mu-1}}{\Gamma(\mu-\lambda)} \sum_{k=0}^{\infty} \frac{\prod_{i=1}^{m} \Gamma\left(a_{i}+\alpha_{i} k\right)}{\prod_{j=1}^{n} \Gamma\left(b_{j}+\beta_{j} k\right)} B_{p, q}(\lambda+k, \mu-\lambda) \frac{z^{k}}{k !},
$$

where ${ }_{\mathrm{p}} \Psi_{\mathrm{q}}(z)$ denotes the Fox-Wright function defined by (see [4, pp. 56-58])

$$
{ }_{m} \Psi_{n}(z)={ }_{m} \Psi_{n}\left[z \mid \begin{array}{c}
\left(a_{i}, \alpha_{i}\right)_{1, m} \\
\left(b_{j}, \beta_{j}\right){ }_{1, n}
\end{array}\right]:=\sum_{k=0}^{\infty} \frac{\prod_{i=1}^{m} \Gamma\left(a_{i}+\alpha_{i} k\right)}{\prod_{j=1}^{n} \Gamma\left(b_{j}+\beta_{j} k\right)} \frac{z^{k}}{k !} .
$$

Proof. Applying the result reported in Theorem 3.2 to the (5.4) and by using the same process as in Theorem 5.5, we report the desired result.

Remark 5.8. If we set $\mathrm{p}=\mathrm{q}$ in (5.3), we re-obtain the interesting known result of Sharma and Devi [17, $\mathrm{p}$. 49, Theorem 8].

\section{Conclusion}

The fractional derivative operator $\mathcal{D}_{z}^{\mu, p, q}\{f(z)\}$ in (3.1) is defined for $\{\mathfrak{R}(p), \mathfrak{R}(q)\} \geqslant 0$. The extended fractional derivatives for the some elementary functions are given by Theorems 3.2-3.6. The Mellin transform of the (3.1) and generating relations of linear and bilinear type for the extended hypergeometric functions are given by Theorems 5.1 to 5.3 , respectively.

\section{Acknowledgment}

The authors extend their appreciation to the International Scientific Partnership Program ISPP at King Saud University for funding this research work through ISPP\# 63.

\section{References}

[1] R. P. Agarwal, P. Agarwal, Extended Caputo fractional derivative operator, Adv. Stud. Contemp. Math., 25 (2015), 301-316. 1

[2] A. Atangana, I. Koca, Chaos in a simple nonlinear system with Atangana-Baleanu derivatives with fractional order, Chaos Solitons Fractals, 89 (2016), 447-454. 1

[3] J.-S. Choi, A. K. Rathie, R. K. Parmar, Extension of extended beta, hypergeometric and confluent hypergeometric functions, Honam Math. J., 36 (2014), 357-385. 1 
[4] A. A. Kilbas, H. M. Srivastava, J. J. Trujillo, Theory and applications of fractional differential equations, North-Holland Mathematics Studies, Elsevier Science B.V., Amsterdam, (2006). 1, 1, 3, 5.4, 5.7

[5] I. Koca, Mathematical modeling of nuclear family and stability analysis, Appl. Math. Sci., 8 (2014), 3385-3392. 1

[6] I. Koca, A method for solving differential equations of q-fractional order, Appl. Math. Comput., 266 (2015), 1-5. 1

[7] M.-J. Luo, G. V. Milovanovic, P. Agarwal, Some results on the extended beta and extended hypergeometric functions, Appl. Math. Comput., 248 (2014), 631-651. 1

[8] F. W. J. Olver, D. W. Lozier, R. F. Boisvert, C. W. Clark (eds.), NIST handbook of mathematical functions, With 1 CD-ROM (Windows, Macintosh and UNIX), U.S. Department of Commerce, National Institute of Standards and Technology, Washington, DC; Cambridge University Press, Cambridge, (2010). 1

[9] N. Ozalp, I. Koca, A fractional order nonlinear dynamical model of interpersonal relationships, Adv. Difference Equ., 2012 (2012), 7 pages. 1

[10] M. A. Özarslan, E. Özergin, Some generating relations for extended hypergeometric functions via generalized fractional derivative operator, Math. Comput. Modelling, 52 (2010), 1825-1833. 1, 2.2

[11] M. A. Özarslan, B. Yilmaz, The extended Mittag-Leffler function and its properties, J. Inequal. Appl., 2014 (2014), 10 pages. 5.6

[12] R. B. Paris, D. Kaminski, Asymptotics and Mellin-Barnes integrals, Encyclopedia of Mathematics and its Applications, Cambridge University Press, Cambridge, (2001). 4

[13] R. K. Parmar, Some generating relations for generalized extended hypergeometric functions involving generalized fractional derivative operator, J. Concr. Appl. Math., 12 (2014), 217-228. 1

[14] R. K. Parmar, T. K. Pogány, Extended Srivastava's triple hypergeometric $\mathrm{H}_{\mathrm{A}, \mathrm{p}, \mathrm{q}}$ function and related bounding inequalities, J. Cont. Math. Anal., (2016). (to appear) 2.2

[15] T. R. Prabhakar, A singular integral equation with a generalized Mittag Leffler function in the kernel, Yokohama Math. J., 19 (1971), 7-15. 5.5

[16] S. G. Samko, A. A. Kilbas, O. I. Marichev, Fractional integrals and derivatives, Theory and applications, Edited and with a foreword by S. M. Nikol'skil, Translated from the 1987 Russian original, Revised by the authors, Gordon and Breach Science Publishers, Yverdon, (1993). 1, 1, 5.4

[17] S. C. Sharma, M. Devi, Certain properties of extended wright generalized hypergeometric function, Ann. Pure Appl. Math. 9 (2014), 45-51. 5.8

[18] H. M. Srivastava, P. W. Karlsson, Multiple Gaussian hypergeometric series, Ellis Horwood Series: Mathematics and its Applications, Ellis Horwood Ltd., Chichester; Halsted Press [John Wiley \& Sons, Inc.], New York, (1985). 1, $2.2,3,5.4$

[19] H. M. Srivastava, H. L. Manocha, A treatise on generating functions, Ellis Horwood Series: Mathematics and its Applications, Ellis Horwood Ltd., Chichester; Halsted Press [John Wiley \& Sons, Inc.], New York, (1984). 1, 3, 5.4

[20] H. M. Srivastava, R. K. Parmar, P. Chopra, A class of extended fractional derivative operators and associated generating relations involving hypergeometric functions, Axioms, 1 (2012), 238-258. 1 